Pacific Journal of Mathematic 


\title{
FOCAL POINTS IN A CONTROL PROBLEM
}

\author{
E. Y. MiKaMI
}

This paper applies a few results on quadratic forms in Hilbert space and the theory of focal points from a paper by Hestenes to a linear control problem with a constraint equation. The abnormality inherent in this problem allows focal intervals to exist. The main results are, after assuming the strengthened Clebsch condition, the following: (1) The signature is equal to the sum of the focal points on the open interval, (2) The focal points are the discontinuous points of rank and abnormality of the conjugate base matrix, and (3) The dimension of a maximal linear space of broken transversal extremal arcs is less than or equal to $n-a$, where $a$ is the abnormality of the problem.

The theory of focal points in the calculus of variations arises in the study of the second variation. It is an accounting of the zeros of the solutions of the Euler-Lagrange equations subject to boundary conditions. The number of zeros is equal to the signature, i.e., the number of negative terms in the associated quadratic form. It is also equal to the number of negative characteristic values of the associated boundary value problem.

The results of this paper were proved earlier by Hazard [2] using calculus of variations methods; the author of this paper approaches a control problem of the same sort by using Hilbert space results from a paper by Hestenes [3]. Earlier work on focal point theory can be found in Birkhoff and Hestenes [1] and Morse [5].

Our problem is the study of the focal points of the quadratic form

$$
J(x)=b^{*} F b+\int_{t^{0}}^{t 1} 2 \omega(t, x, u) d t
$$

where

$$
2 \omega(t, x, u)=x^{*} P(t) x+x^{*} Q(t) u+u^{*} Q^{*}(t) x+u^{*} R(t) u,
$$

relative to a one-parameter family $\mathscr{C}(\lambda)\left(t^{0} \leqq \lambda \leqq t^{1}\right)$ of subspaces of ares satisfying the linear control equation

$$
\dot{x}=A x+B u \quad\left(t^{0} \leqq t \leqq \lambda\right)
$$

a linear constraint equation

$$
M x+N u=0 \quad\left(t^{0} \leqq t \leqq \lambda\right)
$$

and the boundary conditions 


$$
x\left(t^{0}\right)=C b
$$

$$
x(t)=0, u(t)=0
$$

The matrices $A, P=P^{*}$ and $Q$ are square-integrable, $B, M, N$ and $R=R^{*}$ are essentially bounded and measurable, and $C, D$ and $F=F^{*}$ are constant matrices. The matrix $N$ in addition is assumed to have the inverse of $N N^{*}$ existing and essentially bounded. Stated in another way, there exists a positive number $h$ such that at almost all $t$ on $t^{0} \leqq t \leqq t^{1}$,

$$
\pi^{*} N(t) N^{*}(t) \pi \geqq h \pi^{*} \pi
$$

for every $\pi$ in $E^{m}$ (a unitary $m$-dimensional space). An important assumption the strengthened Clebsch condition will be made later.

2. Preliminary remarks. Let $\mathscr{A}$ be a Hilbert space over the complex or real field. A continuous linear functional $L(x)$ on $\mathscr{A}$ is called a linear form and a continuous Hermitian quadratic functional $Q(x)$ on $\mathscr{A}$ is called a quadratic form. The asscciated bilinear form will be denoted by $Q(x, y)$. A quadratic form $K(x)$ is said to be compact on $\mathscr{A}$ if $K\left(x_{q}\right) \rightarrow K\left(x_{0}\right)$ whenever $x_{q}$ converges weakly to $x_{0}$. For any quadratic form $Q(x)$ on $\mathscr{A}$, two vectors $x$ and $y$ are said to be $Q$-orthogonal whenever $Q(x, y)=0$. If $\mathscr{B}$ is a subspace of $A$, then the $Q$-orthogonal complement of $\mathscr{B}$, symbolically $\mathscr{B}^{Q}$, are the vectors in $\mathscr{A} Q$-orthogonal to $\mathscr{B}$. And the space $\mathscr{B}_{0}=\mathscr{B} \cap \mathscr{B}^{Q}$ is called the $Q$-null space; the vectors in $\mathscr{B}_{0}$ are called $Q$-null vectors. If $\mathscr{B}_{0}$ consists only of the null vector, then $Q(x)$ is said to be nondegenerate on $\mathscr{B}$. The relative nullity of $\mathscr{B}$ relative to $\mathscr{A}$, symbolically $r n(\mathscr{B})$, is defined to be the dimension of a linear subspace $\mathscr{C}$ such that $\mathscr{B} 0$ $\mathscr{C}+\mathscr{B} \cap \mathscr{A}_{0}, \mathscr{C} \cap \mathscr{A}_{0}=0$.

The signature (or index) of a quadratic form $Q(x)$ on $\mathscr{A}$, denoted by $s$ or $s(\mathscr{A})$, is the dimension of a maximal linear subspace $\mathscr{B}$ of $\mathscr{A}$ on which $Q(x)$ is negative. The nullity of $Q(x)$ on $\mathscr{A}$, denoted by $n$ or $n(\mathscr{A})$, is the dimension of the $Q$-null space of $\mathscr{A}$. The signature and nullity are well-defined [3]. The quadratic form $J(x)$ in this paper shall have finite signature and nullity because it will be shown to be an elliptic form, i.e., a quadratic form that can be decomposed into a difference $D(x)-K(x)$, where $D(x)$ is a positive definite quadratic form and $K(x)$ is a compact quadratic form.

A quadratic form $Q(x)$ is said to be nonsingular on a linear subspace $\mathscr{B}$ of $\mathscr{A}$ if given a linear form $L(x)$ on $\mathscr{B}$ there is a unique vector $y$ in $\mathscr{B}$ such that $L(x)=Q(x, y)$ for all $x$ in $\mathscr{B}$. A quasinonsingular quadratic form $Q(x)$ on $\mathscr{A}$ is a quadratic form nonsingular on each closed linear subspace on which it is nondegenerate. 
3. The Euler-Lagrange equations. The Hilbert space $\mathscr{H}$ of this paper is composed of vectors of the form

$$
x: x(t), u(t), b\left(t^{0} \leqq t \leqq t^{1}\right),
$$

where $x(t)$ is an $n$-dimensional square-integrable vector, $u(t)$ is a $q$ dimensional square-integrable vector, and $b$ is an $r$-dimensional scalar vector. The inner product is defined to be

$$
(x, y)=c^{*} b+\int_{t_{0}}^{t^{1}}\left[y^{*}(t) x(t)+v^{*}(t) u(t)\right] d t,
$$

where $y: y(t), v(t), c$ is also an element of $\mathscr{X}$.

The one-parameter family $\mathscr{C}(\lambda)\left(t^{0} \leqq \lambda \leqq t^{1}\right)$ described above are subspaces of $\mathscr{H}$ and the quadratic form $J(x)$ is defined for elements $x$ of $\mathscr{H}$ such that $x(t)$ is absolutely continuous.

THEOREM 3.1. Let $x \in \mathscr{H}$ with $x(t)$ absolutely continuous on $t^{0} \leqq t \leqq \lambda$. Then $x$ is J-orthogonal to $\mathscr{C}(\lambda)$ if and only if there exist an absolutely continuous vector $p(t)\left(t^{0} \leqq t \leqq \lambda\right)$ and a squareintegrable vector $\mu(t)\left(t^{0} \leqq t \leqq \lambda\right)$ such that

$$
\begin{array}{cl}
\dot{p}+A^{*} p+M^{*} \mu & =\omega_{x} \\
B^{*} p+N^{*} \mu & =\omega_{u} \\
F b-C^{*} p\left(t^{0}\right) & =0 .
\end{array} \quad\left(t^{0} \leqq t \leqq \lambda\right)
$$

For a proof see Mikami [6].

Equations (3.1), (3.2) together with (1.1), (1.2) shall be called the Euler-Lagrange equations and equation (3.3) is the transversality condition. An element $x$ of $\mathscr{H}$ that satisfies the Euler-Lagrange equations on the subinterval $t^{0} \leqq t \leqq \lambda$ and the transversality condition is called a transversal extremal arc on $t^{0} \leqq t \leqq \lambda$.

4. The strengthened Clebsch condition. Let the symbol $\mathscr{B}$ designate the subspace of $\mathscr{C}$ whose elements satisfy the equations

$$
\dot{x}=A x+B u, M x+N u=0, x\left(t^{0}\right)=C b .
$$

We wish to prove that the quadratic form $J(x)$ is elliptic on $\mathscr{B}$ if and only if the strengthened Clebsch condition holds: There exist positive numbers $h_{0}, h_{1}$ such that at almost all points $t$ on $t^{0} \leqq t \leqq t^{1}$,

$$
\pi^{*} R(t) \pi+h_{1} \pi^{*} N^{*}(t) N(t) \pi \geqq h_{0} \pi^{*} \pi
$$

for all $\pi$ in $E^{q}$. This is equivalent to, as the reader can verify: There exist positive numbers $h_{2}, h_{3}$ such that at almost all $t$ on $t^{0} \leqq t \leqq t^{1}$, 


$$
\pi^{*} R(t) \pi \geqq h_{2} \pi^{*} \pi
$$

whenever $\pi^{*} N^{*}(t) N(t) \pi<h_{3} \pi^{*} \pi$. If the matrices $R(t)$ and $N(t)$ are continuous, then the strengthened Clebsch condition is equivalent to: There exists a positive number $h_{4}$ such that for all $t$ on $t^{0} \leqq t \leqq t^{1}$,

$$
\pi^{*} R(t) \pi \geqq h_{4} \pi^{*} \pi
$$

whenever $N(t) \pi=0$.

Let $\mathscr{A}$ be the subspace of $\mathscr{H}$ whose elements satisfy the equations

$$
\dot{x}=A x+B u, x\left(t^{0}\right)=C b .
$$

The following lemma describes weak convergence in $\mathscr{A}$.

LEMMA 4.1. If $\left\{x_{m}\right\}(m=1,2, \ldots)$ is a sequence in $\mathscr{A}$ converging weakly to $x_{0}$ in $\mathscr{A}$, then $x_{m}(t) \rightarrow x_{0}(t)$ uniformly on $t^{0} \leqq t \leqq t^{1}$.

The proof is an application of the well-known characterization of weak convergence in $L_{2}(n)$.

Define

$$
S(x)=b^{*} b+\int_{t^{0}}^{t^{1}} u^{*} R u d t
$$

Then $S(x)$ is a positive definite quadratic form on $\mathscr{A}$ if and only if the strengthened Legendre condition holds, i.e., there exists a positive number $h_{5}$ such that $\pi^{*} R(t) \pi \geqq h_{5} \pi^{*} \pi$ a.e. on $t^{0} \leqq t \leqq t^{1}$ for all $\pi \in E^{q}$. For a proof see [3]. And one can easily show that

$$
K(x)=\int_{t^{0}}^{t^{1}}\left(x^{*} P x+x^{*} Q u+u^{*} Q^{*} x\right) d t
$$

is a compact quadratic form on $\mathscr{A}$. We summarize these facts as

LEMMA 4.2. The quadratic form $J(x)$ is elliptic on $\mathscr{A}$ if and only if the strengthened Legendre condition holds.

Let $H(x)$ be a quadratic form on $\mathscr{A}$ defined by

$$
H(x)=\int_{t^{0}}^{t^{1}}(M x+N u)^{*}(M x+N u) d t .
$$

THEOREM 4.3. If the strengthened Clebsch condition holds, then $J(x)$ is elliptic on $\mathscr{B}$.

Proof. By Lemma 4.2, $J(x)+h_{1} H(x)$ is elliptic on $\mathscr{A}$, and since $H(x)=0$ on $\mathscr{B}, J(x)$ is elliptic on $\mathscr{B}$. 
To prove the converse of Theorem 4.3 , we begin with

LEMmA 4.4. The quadratic form $H(x)$ is nonnegative and quasinonsingular on $\mathscr{x}$.

Proof. We will utilize a theorem from Hestenes [3, p. 555] which states that a nonnegative quadratic form $Q(x)$ on an arbitrary Hilbert space $\mathscr{C}$ is quasi-nonsingular if and only if it is positive definite on the orthogonal complement of the $Q$-null space $\mathscr{C}_{0}$.

Augment the matrices $M$ and $N$ so that

$$
Y=\left[\begin{array}{c}
M \\
O
\end{array}\right], Z=\left[\begin{array}{c}
N \\
L
\end{array}\right]
$$

are square matrices having $Z^{-1}(t)$ existing a.e. and essentially bounded on $t^{0} \leqq t \leqq t^{1}$. Then

$$
D(x, y)=c^{*} b+\int_{t^{0}}^{t^{1}}(Y y+Z v)^{*}(Y x+Z u) d t
$$

can be shown to be an inner product on $\mathscr{A}$. We wish to show that $H(x)=D(x)$ on the $D$-orthogonal complement of $\mathscr{B}$. For arbitrary vectors $c$ in $E^{r}$ and $w(t)$ in $L_{2}(q-m)$ (if $q=m$, omit $w(t)$ ), there exists a vector $y$ in $\mathscr{B}$ such that

$$
M y+N v=0, L v=w, y\left(t^{0}\right)=C c .
$$

Since $D(x, y)=0$, we have

$$
c^{*} b+\int_{t^{0}}^{t^{1}} w^{*} L u d t=0
$$

and so $b=0$ and $L u=0$. Thus $D(x)=H(x)$ on $\mathscr{B}^{D}$. Observe that $\mathscr{B}$ is the $H$-null space of $\mathscr{A}$ and so in view of the theorem mentioned earlier, $H(x)$ is quasi-nonsingular on $\mathscr{A}$.

THEOREM 4.5. If $J(x)$ is elliptic on $\mathscr{B}$ then the strengthened Clebsch condition holds.

Proof. The quadratic form $H(x)$ is nonnegative and quasi-nonsingular on $\mathscr{A}$ and $J(x)$ is elliptic on $\mathscr{B}$, the $H$-null space of $\mathscr{A}$. A theorem of Hestenes [3, p. 531] asserts the existence of a positive number $s$ such that $J(x)+s H(x)$ is elliptic on $\mathscr{A}$. By Lemma 4.2 this is equivalent to the strengthened Clebsch condition.

Let us define a nonsingularity condition as follows: The inverse of the matrix

$$
R_{1}=\left[\begin{array}{cc}
R & -N^{*} \\
N & 0
\end{array}\right]
$$


exists a.e. on $t^{0} \leqq t \leqq t^{1}$ and has essentially bounded elements. We will prove that the nonsingularity condition holds when the strengthened Clebsch condition is assumed.

LEMMA 4.6. If the strengthened Clebsch condition holds, then there exists a positive number $h_{6}$ such that for almost all $t$ on $t^{0} \leqq$ $t \leqq t^{1}$

$$
\pi^{*}\left(R^{2}+c^{2} N^{*} N\right) \pi \geqq h_{6} \pi^{*} \pi
$$

for all $c \geqq 1$ and all $\pi$ in $E^{q}$.

Proof. Let $I$ be a measureable set of almost all points of $t^{0} \leqq t \leqq t^{1}$ such that if $t \in I$, then $\pi^{*}\left(R(t) \pi \geqq h_{2} \pi^{*} \pi\right.$ whenever

$$
\pi^{*} N^{*}(t) N(t) \pi<h_{3} \pi^{*} \pi
$$

Suppose for each integer $n$, there exists $t_{n} \in I, \pi_{n}{ }^{*} \pi_{n}=1$, such that

$$
\pi_{n}^{*}\left(R^{2}\left(t_{n}\right)+N^{*}\left(t_{n}\right) N\left(t_{n}\right)\right) \pi_{n}<1 / n
$$

So for all $n$ large enough,

Thus

$$
\begin{aligned}
& \pi_{n}^{*} N^{*}\left(t_{n}\right) N\left(t_{n}\right) \pi_{n}<h_{3} \\
& \pi_{n}^{*} R^{2}\left(t_{n}\right) \pi_{n}<h_{2}^{2} .
\end{aligned}
$$

$$
h_{2}^{2}>\pi_{n}^{*} R^{2}\left(t_{n}\right) \pi_{n} \geqq\left(\pi_{n}^{*} R\left(t_{n}\right) \pi_{n}\right)^{2} \geqq h_{2}^{2},
$$

a contradiction.

THEOREM 4.7. If the strengthened Clebsch condition holds, then the nonsingularity condition holds.

Proof. Let

$$
R_{c}=\left[\begin{array}{cc}
R & -c N^{*} \\
c N & 0
\end{array}\right],
$$

We wish to show that $R_{c}^{*} R_{c}$ is positive definite a.e. on $t^{0} \leqq t \leqq t^{1}$ for some number $c$ large enough. So let $w \in E^{q}, z \in E^{m}$ be arbitrary vectors. Then

$$
\left[\begin{array}{ll}
w & z
\end{array}\right]^{*} R_{c}^{*} R_{c}\left[\begin{array}{c}
w \\
z
\end{array}\right] \geqq h_{6}|w|^{2}-2 c k|w \| z|+c^{2} h|z|^{2}
$$

where $k$ is a scalar constant. By choosing $c$ large enough, $R_{c}^{*} R_{c}$ is positive definite. The determinant of $R_{c}$ is bounded away from zero and since 


$$
\left|\operatorname{det} R_{c}\right|=c^{2 m}\left|\operatorname{det} R_{1}\right|,
$$

so is the determinant of $R_{1}$.

5. Abnormality. Let the control equation $\dot{x}=A x+B u$ and the constraint equation $M x+N u=0$ be given. With respect to these equations, there is inherent the notion of abnormality. For the moment define the order of abnormality of a subinterval $t^{2} \leqq t \leqq t^{3}$ of $t^{0} \leqq$ $t \leqq t^{1}$ as the number of linearly independent solutions $\{p, \mu\}, p(t)$ absolutely continuous and $\mu(t)$ square integrable, of

$$
\begin{aligned}
& \dot{p}+A^{*} p+M^{*} \mu=0 \\
& B^{*} p+N^{*} \mu=0
\end{aligned}
$$

a.e. on $t^{2} \leqq t \leqq t^{3}$. Because of the assumption that the inverse of $N N^{*}$ exists and is essentially bounded, we can write

$$
\mu=-\left(N N^{*}\right)^{-1} N B^{*} p .
$$

Equation (5.1) then reduce to the form

$$
\begin{aligned}
& p+A_{1}^{*} p=0 \\
& B_{1}^{*} p=0,
\end{aligned}
$$

and here we refer the reader to [4]. It follows from (5.2) that the order of abnormality is at most $n$.

Later the order of abnormality will be redefined for the problem on hand. Abnormality is important in this paper because it allows nontrivial solutions of the Euler-Lagrange equations to have $x(t)=0$, $u(t)=0$ on a subinterval of $t^{0} \leqq t \leqq t^{1}$.

6. Focal points. The strengthened Clebsch condition is assumed for the rest of this paper. Hence the quadratic form $J(x)$ is elliptic on $\mathscr{B}$ and on any closed subspace of $\mathscr{B}$.

Let $\mathscr{F}$ be the subspace of arcs $x$ in $\mathscr{B}$ that satisfy the EulerLagrange equations and the transversality condition. Such arcs shall be called focal ares. If a focal arc $x$ has $x(t)=0$ on a subinterval $I$ of $t^{0} \leqq t \leqq t^{1}$ then $v(t)=0$ on $I$ also. Now for a fixed $\lambda, t^{0} \leqq \lambda \leqq t^{1}$, the space of focal arcs $\mathscr{F}$ can be decomposed into a direct sum of two subspaces: one that has $x(\lambda)=0$ and one that doesn't. The former subspace can be further decomposed into a direct sum of three subspaces: one that has $x(t)$ vanishing on $t^{0} \leqq t \leqq \lambda$, one that has $x(t)$ vanishing on some subinterval $\lambda \leqq t \leqq \delta, \lambda<\delta$, and one that does not have a combination of these two properties. If the last subspace is denoted by $\mathscr{F}(\lambda)$, then $\mathscr{F}(\lambda)$ is a maximal subspace in $\mathscr{F}$ whose elements $x$ have $x(\lambda)=0$ and $x$ is not a linear combination of arcs $y$ 
that have $y(t)=0$ on $t^{0} \leqq t \leqq \lambda$ and arcs $z$ that have $z(t)=0$ on some $\lambda \leqq t \leqq \delta, \lambda<\delta$. It is the dimension of the subspace $\mathscr{F}(\lambda)$ that interests us.

Let

$$
\mathscr{C}=\left\{x \in \mathscr{B}: x\left(t^{1}\right)=0\right\}
$$

Now a one-parameter family of subspaces $\mathscr{C}(\lambda)\left(t^{0} \leqq \lambda \leqq t^{1}\right)$, which we shall call a resolution of $\mathscr{C}$, has been defined earlier. Let $s(\lambda)$ and $n(\lambda)$ denote the signature and nullity of $J(x)$ on $\mathscr{C}(\lambda)$. The signature is a monotonic nondecreasing function of $\lambda$. A result of Hestenes [3] states that $s(\lambda)=s(\lambda-0)$ and that the dimension of $\mathscr{F}(\lambda)$ is equal to $s(\lambda+0)-s(\lambda)$, i.e., the "jump" at a discontinuity of $s(\lambda)$. So let us define a focal point as a point $\lambda\left(t^{0} \leqq \lambda<t^{1}\right)$ such that $\mathscr{F}(\lambda)$ is not the null space and define the order of the focal point $\lambda$ as the dimension of $\mathscr{F}(\lambda)$. In other words $\lambda$ is a focal point if and only if there exists a focal arc $x \neq 0$ such that $x(t)$ vanishes at $\lambda$ and $x$ is not a linear sum of arcs that have $y(t)=0$ on $t^{0} \leqq$ $t \leqq \lambda$ and arcs that have $z(t)=0$ on some $\lambda<t \leqq \delta$. Later we shall refer a focal point as a focal point of the end point, for example, $\lambda$ is a focal point of $t^{0}$ in the above case.

In the special case where the matrix $C=0$, i.e., $x\left(t^{0}\right)=0$, the word "conjugate" is used instead of "focal".

The above facts can be summarized in the following:

THEOREM 6.1. The signature of $J(x)$ on $\mathscr{C}$ is equal to the sum of the order of the focal points $\lambda, t^{0}<\lambda<t^{1}$.

Theorem 6.1 was proved by Birkhoff and Hestenes [1] for the case $\dot{x}=u$ and by Hazard [2] for the case $\dot{x}=u, M x+N u=0$. Hazard used focal intervals, i.e., intervals on which a focal are vanishes. Our focal point is the right endpoint of Hazard's focal interval.

We have described the notion of abnormality, relating it to the existence of focal intervals. For example, if the problem were normal, i.e., the order of abnormality equals zero for all subintervals of $t^{0} \leqq$ $t \leqq t^{1}$, then the focal intervals collapse into points. In fact if the order of abnormality is the same for all subintervals, then again the focal intervals collapse into points.

7. Conjugate bases. In a simple variational problem, for example, one in which the arcs satisfy

$$
\dot{x}=u, b=0, x\left(t^{0}\right)=0, x\left(t^{1}\right)=0,
$$

and the strengthened Legendre condition holds, it is well-known that 
there exists a conjugate base of the left end point $t^{0}, x_{\alpha}(t) \quad(\alpha=$ $1, \cdots, n)$, such that the zeros of the determinant of the matrix

$$
\left(x_{\alpha}^{i}(t)\right)
$$$$
\left(t^{0}<t<t^{1} ; i, \alpha=1, \cdots, n\right)
$$

are the conjugate points of $t^{0}$ and that the order of a conjugate point $\lambda, t^{0}<\lambda<t^{1}$, is equal to $n$ minus the rank $\left(x_{\alpha}^{i}(\lambda)\right)$. In our control problem the determinant of the matrix (7.1) would be zero on focal intervals and even possibly identically zero. In short the determinant does not always give the focal points; however, the rank and the abnormality together provide the location of the focal points.

Let us begin by assuming that the matrix $\left[F, C^{*}\right]$ has full rank $r$. And let us define a focal extremal (relative to $t^{\circ}$ ) to be a system of vectors

$$
x: x(t), u(t), b, p(t), \mu(t) \quad\left(t^{0} \leqq t \leqq t^{1}\right)
$$

satisfying the Euler-Lagrange equations, the end condition (1.3), and the transversality condition. It follows easily that if $x$ and $y$ are focal extremals then

$$
\frac{d}{d t}\left(p^{*} y-x^{*} q\right)=0
$$

So we say that two focal extremals are conjugate to each other if

$$
p^{*}(t) y(t)-x^{*}(t) q(t)=0 \quad\left(t^{0} \leqq t \leqq t^{1}\right) .
$$

THEOREM 7.1. There exists a linearly independent set of mutually conjugate focal extremals, $x_{\alpha}(\alpha=1, \cdots, n)$, such that every focal extremal can be written as a finite linear combination of the $x_{\alpha}$.

The proof is a simple exercise in differential equation theory.

The set described in Theorem 7.1 shall be called a conjugate base (of $t^{0}$ ).

\section{THEOREM 7.2. Let}

$$
x_{\alpha}: x_{\alpha}(t), u_{\alpha}(t), b_{\alpha}, p_{\alpha}(t), \mu_{\alpha}(t) \quad\left(t^{0} \leqq t \leqq t^{1} ; \alpha=1, \cdots, n\right)
$$

be a set of linearly independent mutually conjugate vectors satisfying the Euler-Lagrange equations and having $a_{\alpha} x_{\alpha}\left(t^{0}\right)=0$ (summed over $\alpha)$ whenever $a_{\alpha} b_{\alpha}=0$ ( $a_{\alpha}$ are scalars). Then there exist matrices $C$ and $F$ such that $F=F^{*}$, rank $\left[F, C^{*}\right]=r, x_{\alpha}\left(t^{0}\right)=C b_{\alpha}$, and

$$
F b_{\alpha}-C^{*} P_{\alpha}\left(t^{0}\right)=0 \text {. }
$$

Proof. Without loss of generality assume 


$$
\begin{aligned}
& b_{1}=[10 \cdots 0]^{*} \\
& \cdots \\
& b_{r}=[0 \cdots 010 \cdots 0]^{*}(1 \text { in } \gamma \text {-th position }) \\
& b_{r+1}=\cdots=b_{n}=0 .
\end{aligned}
$$

Let the matrix $C$ be the columns of $\left[x_{1}\left(t^{0}\right), \cdots, x_{\gamma}\left(t^{0}\right), 0, \cdots, 0\right]$. Then $x_{\alpha}\left(t^{0}\right)=C b_{\alpha}(\alpha=1, \cdots, n)$.

Let $F$ be the matrix

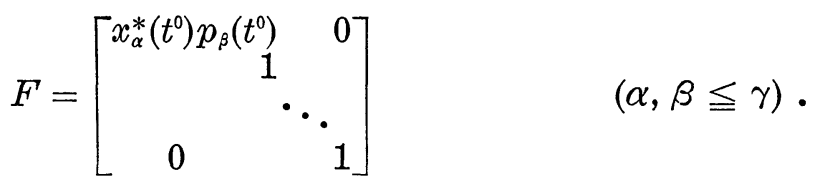

Then $F=F^{*}$ because the $x_{\alpha}$ are mutually conjugate. The remaining relations are easily verified.

Let $t^{0}<\lambda<t^{1}$. The order of abnormality $a(\lambda)$ of this problem is defined to be the number of linearly independent solutions $\{p, \mu, b\}$ of the equations

$$
\begin{aligned}
& \dot{p}+A^{*} p+M^{*} \mu=0 \\
& B^{*} p+N^{*} \mu=0 \\
& C b=0, F b-C^{*} p\left(t^{0}\right)=0 \cdot \quad\left(t^{0} \leqq t \leqq \lambda\right)
\end{aligned}
$$

By referring back to $\S 5$ we see that the order of abnormality $a(\lambda)$ is a nonincreasing function of $\lambda$ and continuous from the left. It is at most $n$.

THEOREM 7.3. If $x_{\alpha}(\alpha=1, \cdots, n)$ is a conjugate base and if $d(t)$ is the rank of the matrix $x_{\alpha}^{i}(t)\left(t^{0} \leqq t \leqq t^{1} ; i, \alpha=1, \cdots, n\right)$, then the focal points of $t^{0}$ are the points $\lambda, t^{0}<\lambda<t^{1}$ at which

$$
d(\lambda+0)+a(\lambda+0)-d(\lambda)-a(\lambda)>0 .
$$

Moreover if $\lambda$ is such a focal point, then the order of the focal point equals the left-hand side of (7.2). The nullity of $J(x)$ on $\mathscr{C}$ is equal to $n-d\left(t^{1}\right)$.

The proof follows from the definitions of a focal point and abnormality.

8. Separated end conditions. This section deals with a subspace having separated end conditions. The control parameter $b$ is composed of two component vectors

$$
b: b_{0}^{g}, b_{1}^{\overline{1}} \quad(\sigma=1, \cdots, r: \tau=1, \cdots, s) .
$$


Let $\mathscr{D}$ denote the vectors $x$ in $\mathscr{H}$ that satisfy

$$
\begin{aligned}
& \dot{x}=A x+B u, M x+N u=0 \\
& x\left(t^{0}\right)=C_{0} b_{0}, x\left(t^{1}\right)=C_{1} b_{1} .
\end{aligned}
$$

Let

$$
\begin{aligned}
\mathscr{C}(\lambda)=\left\{x \in \mathscr{D}: b_{1}=0, x(t)=0, u(t)=0 \text { on } \lambda \leqq t \leqq t^{1}\right\} \\
\mathscr{D}(\lambda)=\left\{x \in \mathscr{D}: b_{0}=0, x(t)=0, u(t)=0 \text { on } t^{0} \leqq t \leqq \lambda\right\} \\
\left(t^{0} \leqq \lambda \leqq t^{1}\right)
\end{aligned}
$$

be resolutions of $\mathscr{C}\left(t^{1}\right)$ and $\mathscr{D}\left(t^{0}\right)$, respectively. The quadratic form $J(x)$ is written

$$
J(x)=b_{0}^{*} F_{0} b_{0}+b_{1}^{*} F_{1} b_{1}+\int_{t^{0}}^{t^{1}} 2 \omega(t, x, u) d t,
$$

where $F_{0}=F_{0}^{*}$ and $F_{1}=F_{1}^{*}$. Again assume

$$
\operatorname{rank}\left[F_{0}, C_{0}^{*}\right]=r, \operatorname{rank}\left[F_{1}, C_{1}^{*}\right]=s .
$$

Let $t^{0}<\lambda<t^{1}$. For convenience let

$$
\mathscr{E}(\lambda)=[\mathscr{C}(\lambda)+\mathscr{D}(\lambda)]^{J}=\mathscr{C}(\lambda)^{J} \cap \mathscr{D}(\lambda)^{J}
$$

( $J$-orthogonality relative to $\mathscr{D}$ ). An arc $x$ is in $\mathscr{E}(\lambda)$ if and only if $x$ is a transversal extremal arc on $t^{0} \leqq t \leqq \lambda$ and a transversal extremal arc on $\lambda \leqq t \leqq t^{1}$. If the vector $p(t)$ has a discontinuity at $\lambda$, then we say that $x$ is a broken transversal extremal arc. A result of $[3, \mathrm{p} .565]$ is

$$
s(\mathscr{D})=s[\mathscr{C}(\lambda)]+s[\mathscr{D}(\lambda)]+k(\lambda)
$$

where

$$
k(\lambda)=s[\mathscr{E}(\lambda)]+r n[\mathscr{C}(\lambda)+\mathscr{D}(\lambda)] .
$$

The number $k(\lambda)$ is the dimension of a maximal linear space of broken transversal extremal arcs having $J(x) \leqq 0$. Note that $k(\lambda)$ is constant except at focal points of $t^{0}$ or $t^{1}$ by virtue of (8.1).

In normal problems it is known that $k(\lambda) \leqq n$. This is also true for problems with the order of abnormality not equal to zero. Let $a$ be the maximal number of linearly independent solutions $\left\{p, \mu, b_{0}\right.$, $b_{1}$ \} of the equations

$$
\begin{array}{ll}
\dot{p}+A^{*} p+M^{*} \mu=0 & \\
B^{*} p+N^{*} \mu=0 & \left(t^{0} \leqq t \leqq t^{1}\right) \\
F_{1} b_{1}+C_{1}^{*} p\left(t^{1}\right)=0 & \\
F_{0} b_{0}-C_{0}^{*} p\left(t^{0}\right)=0 & \\
C_{1} b_{1}=0, C_{0} b_{0}=0 . &
\end{array}
$$


We call the number $a$ the order of abnormality of the problem.

THEOREM 8.1.

$$
\begin{array}{cl}
0 \leqq k(\lambda) \leqq n-a & \left(t^{0}<\lambda<t^{1}\right) \\
s(\mathscr{D}) & =s\left(\mathscr{D}\left(t^{0}\right)\right)+k\left(t^{0}+0\right) \\
s(\mathscr{D}) & =s\left(\mathscr{C}\left(t^{1}\right)\right)+k\left(t^{1}-0\right)
\end{array}
$$

Proof. Let $\left\{p_{\alpha}, \mu_{\alpha}, b_{0 \alpha}, b_{1 \alpha}\right\}(\alpha=1, \cdots, a)$ be linearly independent solutions of (8.2)-(8.3). Fix $t^{0}<\lambda<t^{1}$ and abbreviate

$$
e=\operatorname{rn}[\mathscr{C}(\lambda)+\mathscr{D}(\lambda)] \text {. }
$$

Then there exist $J$-null vectors $x_{\beta}$ of $\mathscr{C}(\lambda)+\mathscr{D}(\lambda)$ and vectors $p_{\beta}$ $(\beta=1, \cdots, e)$ such that $x_{\beta}(\lambda)=0$ and the set of vectors

$$
p_{\alpha}(\lambda), p_{\beta}(\lambda-0)-p_{\beta}(\lambda+0) \quad(\alpha=1, \cdots, a ; \beta=1, \cdots, e)
$$

are linearly independent. If not, then there would exist a vector $x$ equal to a finite linear combination of the $x_{\beta}$ and a vector $p$ continuous at $\lambda$, a contradiction. Since the vectors $x_{\beta}$ are $J$-orthogonal to $\mathscr{E}(\lambda)$ it can be verified by computation that

$$
\begin{array}{cc}
p_{\alpha}(\lambda)^{*} y(\lambda)=0 & (\alpha=1, \cdots, a) \\
{\left[p_{\beta}(\lambda-0)-p_{\beta}(\lambda+0)\right]^{*} y(\lambda)=0} & (\beta=1, \cdots, e)
\end{array}
$$

for each $y$ in $\mathscr{E}(\lambda)$. This means there are $n-a-e$ linearly independent $y(\lambda)$ and therefore the number of linearly independent vectors in $\mathscr{E}(\lambda)$ whose linear combinations do not vanish at $\lambda$ is less than or equal to $n-a-e$. Since the vectors in $\mathscr{E}(\lambda)$ that have $y(\lambda)=0$ have $J(y)=0$, we conclude that $s[\mathscr{E}(\lambda)] \leqq n-a-e$. Finally $k(\lambda) \leqq$ $(n-a-e)+e=n-a$.

To prove (8.5) choose $\lambda$ in (8.1) near $t^{0}$ so that no focal point of $t^{\circ}$ or $t^{1}$ is in $t^{0}<t \leqq \lambda$. Equation (8.5) follows readily.

COROLlary 8.2. If $s_{0}$ and $s_{1}$ respectively denote the number of focal points of $t^{0}$ and $t^{1}$ on a given interval $I$, then the following relations hold:

$$
\begin{aligned}
& s_{0}-s_{1}=k\left(t^{0}+0\right)-k\left(t^{1}-0\right) \text { if } I=\left(t^{0}, t^{1}\right) \\
& s_{1}-s_{1}=k\left(t^{0}+0\right)-k\left(t^{1}+0\right) \text { if } I=\left(t^{0}, t^{1}\right] \\
& s_{0}-s_{1}=k\left(t^{0}-0\right)-k\left(t^{1}-0\right) \text { if } I=\left[t^{0}, t^{1}\right) \\
& s_{0}-s_{1}=k\left(t^{0}-0\right)=k\left(t^{1}+0\right) \text { if } I=\left[t^{0}, t^{2}\right] .
\end{aligned}
$$

In each case $\left|s_{0}-s_{1}\right| \leqq n-a$. 


\section{REFERENCES}

1. G. D. Birkhoff and M. R. Hestenes, Natural isoperimetric conditions in the calculus of variations, Duke Math. J. 1 (1935), 198-286.

2. K. E. Hazard, Index theorems for the problem of Bolza in the calculus of variations, Contributions to the Calculus of Variations 1938-1941, The University of Chicago Press, 293-356.

3. M. R. Hestenes, Applications of the theory of quadratic forms in Hilbert space to the calculus of variations, Pacific J. Math. 1 (1951), 525-581.

4. — Calculus of Variations and Optimal Control Theory, John Wiley and Sons, New York, 1966.

5. M. Morse, The Calculus of Variations in the Large, American Math. Society Colloquium Publications Vol. 18, New York, 1934.

6. E. Y. Mikami, Quadratic optimal control problems, UCLA doctoral dissertation, 1968.

Received November 19, 1969.

MiCHELSON LABORATORIES

Naval Weapons Center

China lake, California 



\section{PACIFIC JOURNAL OF MATHEMATICS}

\section{EDITORS}

\author{
H. SAMELSON \\ Stanford University \\ Stanford, California 94305

\section{Richard Pierce} \\ University of Washington \\ Seattle, Washington 98105
}

J. DugundJI

Department of Mathematics

University of Southern California

Los Angeles, California 90007

RichaRd ARENS

University of California

Los Angeles, California 90024

\section{ASSOCIATE EDITORS}
E. F. BECKENBACH
B. H. NeUMANN
F. WOLE
K. YoshidA

\section{SUPPORTING INSTITUTIONS}

\author{
UNIVERSITY OF BRITISH COLUMBIA \\ CALIFORNIA INSTITUTE OF TECHNOLOGY \\ UNIVERSITY OF CALIFORNIA \\ MONTANA STATE UNIVERSITY \\ UNIVERSITY OF NEVADA \\ NEW MEXICO STATE UNIVERSITY \\ OREGON STATE UNIVERSITY \\ UNIVERSITY OF OREGON \\ OSAKA UNIVERSITY \\ UNIVERSITY OF SOUTHERN CALIFORNIA
}

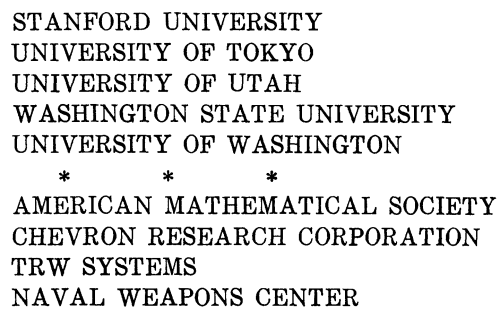

The Supporting Institutions listed above contribute to the cost of publication of this Journal, but they are not owners or publishers and have no responsibility for its content or policies.

Mathematical papers intended for publication in the Pacific Journal of Mathematics should be in typed form or offset-reproduced, (not dittoed), double spaced with large margins. Underline Greek letters in red, German in green, and script in blue. The first paragraph or two must be capable of being used separately as a synopsis of the entire paper. The editorial "we" must not be used in the synopsis, and items of the bibliography should not be cited there unless absolutely necessary, in which case they must be identified by author and Journal, rather than by item number. Manuscripts, in duplicate if possible, may be sent to any one of the four editors. Please classify according to the scheme of Math. Rev. Index to Vol. 39. All other communications to the editors should be addressed to the managing editor, Richard Arens, University of California, Los Angeles, California, 90024.

50 reprints are provided free for each article; additional copies may be obtained at cost in multiples of 50 .

The Pacific Journal of Mathematics is published monthly. Effective with Volume 16 the price per volume (3 numbers) is $\$ 8.00$; single issues, $\$ 3.00$. Special price for current issues to individual faculty members of supporting institutions and to individual members of the American Mathematical Society: $\$ 4.00$ per volume; single issues $\$ 1.50$. Back numbers are available.

Subscriptions, orders for back numbers, and changes of address should be sent to Pacific Journal of Mathematics, 103 Highland Boulevard, Berkeley, California, 94708.

PUBLISHED BY PACIFIC JOURNAL OF MATHEMATICS, A NON-PROFIT CORPORATION

Printed at Kokusai Bunken Insatsusha (International Academic Printing Co., Ltd.), 7-17, Fuj̣imi 2-chome, Chiyoda-ku, Tokyo, Japan. 


\section{Pacific Journal of Mathematics}

\section{Vol. 35, No. $2 \quad$ October, 1970}

Valentin Danilovich Belousov and Palaniappan L. Kannappan, Generalized Bol functional equation .................................... 259

Charles Morgan Biles, Gelfand and Wallman-type compactifications ........... 267

Louis Harvey Blake, A generalization of martingales and two consequent convergence theorems .................................... 279

Dennis K. Burke, On p-spaces and $w \Delta$-spaces..................... 285

John Ben Butler, Jr., Almost smooth perturbations of self-adjoint operators . . . . . . 297

Michael James Cambern, Isomorphisms of $C_{0}(Y)$ onto $C(X) \ldots \ldots \ldots \ldots \ldots . \ldots 307$

David Edwin Cook, A conditionally compact point set with noncompact closure ... 313

Timothy Edwin Cramer, Countable Boolean algebras as subalgebras and homomorphs .........................................

John R. Edwards and Stanley G. Wayment, A v-integral representation for linear operators on spaces of continuous functions with values in topological vector spaces.............................................

Mary Rodriguez Embry, Similarities involving normal operators on Hilbert

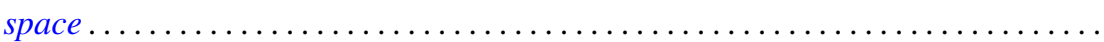

Lynn Harry Erbe, Oscillation theorems for second order linear differential

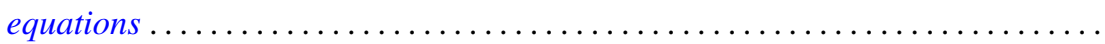

William James Firey, Local behaviour of area functions of convex bodies .......... Joe Wayne Fisher, The primary decomposition theory for modules ..............

Gerald Seymour Garfinkel, Generic splitting algebras for Pic ..................

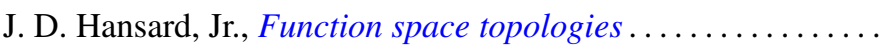

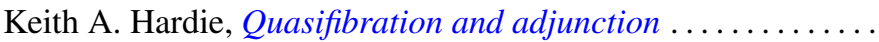

G. Hochschild, Coverings of pro-affine algebraic groups ...........

Gerald L. Itzkowitz, On nets of contractive maps in uniform spaces ..

381

389

399

417

Melven Robert Krom and Myren Laurance Krom, Groups with free nonabelian subgroups....................................

James Robert Kuttler, Upper and lower bounds for eigenvalues by finite differences ......................................

Dany Leviatan, A new approach to representation theory for convolution transforms . . .

Richard Beech Mansfield, Perfect subsets of definable sets of real numbers ...

Brenda MacGibbon, A necessary and sufficient condition for the embedding of a

Lindelof space in a Hausdorff $\mathscr{H} \sigma$ space ..................

David G. Mead and B. D. McLemore, Ritt's question on the Wronskian ....

Edward Yoshio Mikami, Focal points in a control problem .....

Paul G. Miller, Characterizing the distributions of three independent n-dimensional random variables, $X_{1}, X_{2}, X_{3}$, having analytic characteristic functions by the joint distribution of $\left(X_{1}+X_{3}, X_{2}+X_{3}\right)$. . .

P. Rosenthal, On the Bergman integral operator for an elliptic partial differential equation with a singular coefficient....

Douglas B. Smith, On the number of finitely generated $O$-group 